\title{
Fracturas diafisiarias DE
}

\section{ANTEBRAZO: CARACTERISTICAS RADIOLOGICAS EN NIÑOS Y ADOLESCENTES EN HOSPITALES DEL MINSA-CUSCO 1995-2001}

\author{
Autores: Fredy Juvenal Huallpa Jordán"*, Fredy Quispe Tocre ${ }^{* *}$ \\ Coautor: Gary Raúl Molero Tejeira**
}

RESUMEN:

Palabras clave:

\begin{abstract}
Se presenta un trabajo de investigación descriptivo, retrospectivo y transversal que planted como problema: ¿Cuál es el grado de angulación y porcentaje de desplazamiento en las fracturas diafisiarias de antebrazo luego de la reducción incruenta en pacientes con edad entre tres y diecisiete años entre 1995 y el 2001 en los hospitales del MINSA del Cusco?. Entre las variables se hallan: angulación, desplazamiento, sexo, edad, trazo fracturario, antebrazo y hueso fracturado, localización, extensión y dirección de la angulación de la fractura y mecanismo de acción. Los resultados fueron el hallazgo de 217 pacientes. Las características halla das fueron. sexo masculino, mecanismo indirecto, afectación de ambos huesos, dirección de angulación varoventral, extensión completa, trazo transversal y localización en el tercio infe. rior. Las características de la angulación y el desplazamiento después de la reducción incruen. ta fueron: angulación del cúbito en la incidencia de frente una media de 4,7 grados y en la de perfil 4,2 grados. Angulación de radio en la incidencia de frente una media de 10,71 grados y en la de perfil 10,09 grados. El promedio de desplazamiento para ambos huesos logrado luego del tratamiento fue de $21,43 \%$ para el cúbito y $24,83 \%$ para el radio. La media encontrada en el cabalgamiento fue de 6,27 mm para el cúbito y $5,06 \mathrm{~mm}$ para el radio. Los casos considera. des como aceptables en el caso de la edad son de 29,03\% y 39,17\% para los grupos de 3 a 5 años y de 10 a 17 años respectivamente. Realizado el análisis inferencial usando como prueba estadística a la chi-cuadrado de McNemar no se halló relación estadísticamente significativa entre edad o sexo y condición de aceptabilidad.

Fractura de antebrazo, radiología.
\end{abstract}

** Médicos egresados de la Facultad de Medicina-UNSAAC. 
SUMMARY:

A descriptive, retrospective and traverse investigation work is presented that outlines as problem: Which is it the angulation grade and displacement percentage in the fractures of forearm after the bloodless reduction in patient with age between three and seventeen years between 1995 and the 2001 in the hospitals of the MINSA of the Cusco?. Among the variables they are: angulation, displacement, sex, age, delineation of the fracture, forearm and fractured bone, localization, extension and address of the angulation of the fracture and action mechanism. The results were the discovery of 217 patients. The characteristics pickups were: masculine sex, indirect mechanism, affectation of both bones, address of angulación varoventral, complete extension, trace traverse and localization in the inferior third: The characteristics of the angulacion and the displacement after the bloodless reduction were: angulation of the ulna in the front incidence a stocking of 4,7 grades and in that of profile 4,2 grades. Angulation of the radius in the front incidence a stocking of 10,71 grades and in that of profile 10,09 grades. The displacement average for both bones achieved after the treatment was of $21,43 \%$ for the ulna and $24,83 \%$ for the radius. The stocking found in the superpossed bones were of $6,27 \mathrm{~mm}$ for the ulna and 5,06 $\mathrm{mm}$ for the radius. The cases considered as acceptable in the case of the age are of $29,03 \%$ and $39,17 \%$ for the groups from 3 to 9 years and of 10 to 17 years respectively. Carried out the analysis using like statistical test to the chi-square of McNemar was not relationship statistically significant between age or sex and acceptability condition.

\section{PROBLEMA}

¿Cuál es el grado de angulación y porcentaje de desplazamiento en las fracturas diafisiarias de antebraz antes y después de la reducción incruenta en pacientes con edad entre tres y diecisiete años entre 1995 y el 2001 en los hospitales del MINSA del Cusco?

\section{OBJETIVOS}

General : Determinar la angulación y desplazamiento en fracturas diafisiarias del antebrazo.

\section{Específicos:}

Medir y comparar la angulación y desplazamiento en fracturas diafisiarias del antebrazo, antes y después de la reducción incruenta, determinar la edad el sexo, la dirección de la angulación, la extensión de la fractura, el tipo de trazo fracturario, la localización, el antebrazo afectado. el hueso o huesos afectados y el mecanismo de acción más frecuente en las fracturas diafisiarias de antebrazo.

\section{MARCO TEORICO}

\section{BASESTEÓRICAS:}

Fracturas de antebrazo.

Las fracturas del antebrazo son aquéllas que comprometen la diáfisis del radio y del cúbito. Se considera, en forma algo arbitraria, que el límite proximal del antebrazo pasa por la tuberosidad bicipital y el distal a $405 \mathrm{~cm}$ de la articulación de la muñeca. La amplitud total del movimiento pronosupinatorio es de $180^{\circ}$ : la cúpula radial esta en el eje del movimiento en la parte proximal, la cubital en la distal. Alrededor de este eje gira el radio sobre el cúbito, el cual esta fijo en su articulación con la tróclea humeral.
Debemos considerar que, cada vez que ocurre la fracura de un hueso del antebrazo, puede existir la fractura del otro o una luxación de las articulaciones radioulnar, ya sea proximal o distal. Las fracturas aisladas de cúbito o radio son más bien infrecuentes. El mayor numero de fracturas de antebrazo ocurre durante la infancia y la adolescencia y es mucho más frecuente en varones que en lescencia y
mujeres
( 3$)$

Blount indica que el $75 \%$ de las fracturas de la diáfisis radial y cubital se localizan en el tercio distal, el $18 \%$ en el tercio medio y el $7 \%$ en el tercio proximal.

Las fracturas diafisiarias del antebrazo se clasifican. según su localización, en fracturas del tercio superior, tercio medio y tercio inferior. Como referencia se utiliza la curvatura radial: el tercio superior termina donde comienza ésta, y el tercio inferior comienza donde finaliza la curva.

Tachdjian clasifica de esta forma: Según la localización en tercio distal, medio o superior. Según los huesos comprometidos en cubital, radial o cubitoradial, Según la solución de continuidad en rama verde o completa que se divide a su vez en fracturas con desplazamiento mínimo o extraordinario con cabalgamiento; para cada hueso. Según su angulación puede ser con dirección palmar, dorsal, hacia el espacio interóseo o alejándose de ella. Según el tipo de fractura, la AO/ASIF recomienda la clasificación: Trazo transversal simple de cada hueso o de ambos, con fragmento en mariposa en cada hueso o de ambos y combinaciones de fracturas completas (conminutas de uno o ambos huesos).

\section{Fisiopatologia de la fractura.}

El mecanismo de producción de estas fracturas es generalmente indirecto en nin̄os, por caída apoyándose con la palma de la mano, fuerza que aumenta la inflexión de las curvaturas normales de las diáfisis radial y cubital produciéndose la fractura, que con mayor frecuencia está 
Fracturas diafisiariasde antebrazo: caracteristicas radiologicas en niños y adolescentes hospitales - MinSa - Cusco

localizada en el tercio medio y distal del antebrazo. El mecanismo directo puede producir una fractura de sólo uno de los dos huesos del antebrazo, siendo éste mecanismo más frecuente en el adulto, por maniobras de de. fensa personal.

Si la fractura se encuentra por sobre la inserción distal del pronador redondo, el fragmento proximal del radio gira en supinación por la acción del biceps y supinador corto, mientras que el fragmento distal gira en pronación por acción del pronador redondo y pronador cuadrado. Si la fractura es distal a la inserción del pronador redondo, el fractura es distal a la inserción del pronador redondo, el que el fragmento distal gira en pronación por acción del pronador cuadrado.

El desplazamiento consiste en la pérdida de contacto de los extremos fracturarios el que si es total produce el cabalgamiento de los segmentos fracturarios del radio y cúbito por la acción de los músculos del antebrazo en el eje longitudinal, y angulación de los segmentos en el foco de fractura (en el eje axial). ${ }^{(3)}$

\section{Clinica}

El cuadro clínico es variable, según el tipo de lesión, pero habitualmente se comprueba una tumefacción edematosa que altera la morfología normal del antebrazo, con o sin equimosis, y dolor exquisito al nivel de la fractura. Cuando existen desplazamientos se hallan angulación de los ejes, ascenso de la apofisis estiloides del radio, desviación radial de la mano (eje del miembro en bayoneta), porción distal del antebrazo en franca pronación, movilidad anormal y crepitación ósea.

Los movimientos de las articulaciones del codo y muñeca estarán limitados, y su movilización pasiva provocará dolor; lo más afectado será la pronosupinación antebraquial.

Tratamiento

En los niños el tratamiento de elección es ortopédico, realizando la reducción seguida de una inmovilización con yeso braquiopalmar. La inmovilización se mantiene por 4 a 6 semanas según la edad del niño y la evolución de la consolidación ósea. En las fracturas en tallo verde de tercio distal del antebrazo, que habitualmente sólo se desplazan hacia dorsal, basta con la corrección de esta angulación seguida de yeso braquiopalmar. Las fracturas del antebrazo pueden reducirse pero son difíciles de contener y no es raro que, lograda una reducción anatómica, ésta se desplace aun dentro del yeso. Por otra parte, la inmovilización prolongada puede llevar a rigideces irreversibles del codo.

Desde el punto de vista funcional se puede aceptar (condición de aceptabilidad) como buena reducción a la corrección del cabalgamiento, el contacto de medio diámetro diafisiario, angulaciones de hasta $10^{\circ}$ y corrección de toda rotación. De lo contrario las articulaciones distales trabajan en desalineamiento o desnivel respecto de 10 normal y sobreviene su artrosis. ${ }^{(4)}$

\section{HIPOTESIS}

El grado de angulación es menor o igual a 10 grados $y$ el porcentaje de desplazamiento es menor o igual a $50 \%$, en fracturas diafisiarias de antebrazo en pacientes con edad entre tres y diecisiete años; luego de la reduc ción incruenta, entre enero de1995 y noviembre del 2001 en los hospitales del MINSA del Cusco.

\section{VARIABLESEINDICADORES.}

- Variables implicadas: Angulación y desplazamiento

- Variables no implicadas intervinientes: De sujeto (sexo, edad, trazo fracturario, antebrazo fracturado, hueso fracturado, localización de la fractura, extensión de la fractura y dirección de la angulación) y de ambiente (mecanismo de acción).

\section{DISEÑO METODOLÓGICO}

Tipo de estudio: Descriptivo, retrospectivo, transversal.

Población o universo: Grupo de estudio: Pacientes con diagnóstico de fractura diafisiaria de antebrazo que acudieron al Hospital Regional del Cusco o al Hospital Antonio Lorena del Cusco entre enero de 1995 y noviembre del 2001.

Muestra: Total de los pacientes que cumplan los criterios de inclusión.

Criterios de inclusión: Todos los pacientes con diagnós tico de fractura diafisiaria de antebrazo en el Hospital Regional del Cusco y Hospital Antonio Lorena del Cusco gional del Cusco y rospital Anto del 2001, cuya edad al entre enero de 1995 y noviembre del 2001, cuya edad al años y pacientes que cuenten con radiografías antes y luego de la reducción incruenta.

Criterios de exclusión: Pacientes con indicación de reducción quirúrgica, pacientes con trazo fracturario diferente a los mencionados en la variable "trazo fracturario", pacientes que no cuenten con alguna de las radiografías y pacientes con diagnóstico de refractura diafisiaria de antebrazo.

Procedimiento de obtención de datos: La obtención de datos se realizará mediante la revisión de historias clínicas de todos los pacientes con diagnostico de fractura diafisiaria de antebrazo y que cumplan los criterios de inclusión y exclusión y revisión de radiografias y su medición.

Fuentes de información: Historias clínicas y radiografias.

Analisis estadistico: Se utilizará el Paquete estadístico SPSS en su versión 9.0, para establecer relaciones entre los resultados de corrección de fracturas con edad y sexo, utilizando la chi-cuadrado de McNemar y cuando se requiera se utilizará la corrección de Yates, adoptando un valor de significancia de $p<0,05$ y una confiabilidad del $95 \%$. 


\section{RESULTADOS Y CONCLUSIONES}

El tipo de trazo fracturario más frecuente fue el transversal en ambos huesos; la fractura se localiza en mayo número en el tercio inferior en ambos huesos, el antebrazo más afectado fue el izquierdo, la afectación simultanea de ambos huesos fue la más frecuente, el mecanismo de acción más frecuente fue el indirecto, la extensión de la fractura predominante fue la completa y la dirección de angulación más frecuente en ambos huesos fue la varoventral.

Los promedios de angulación antes de la reducción incruenta fueron de 11,64 y 10,40 grados en las incidencias de frente y de perfil y luego de la reducción fueron de 4,7 y 4,2 grados en las incidencias de frente y perfil en el cúbito. En el caso del radio de 10,72 grados y 10,09 grados antes de la reducción y de 4,46 y 4,74 grados en las incidencias de frente y perfil respectivamente después de la reducción.

Los promedios de desplazamiento antes de la reducción incruenta fueron de $39,02 \%$ para el cúbito y $44,08 \%$ para el radio. Luego de la reducción estos valores fueron de $21,43 \%$ y $24,83 \%$ para el cúbito y el radio respectivamente.

La comparación de medias de la angulación y el desplazamiento antes y después de la reducción incruenta indica que el procedimiento disminuye los valores a niveles aceptables en la mayoria de los casos. La edad más frecuente afectada fue la de 12 años y el sexo con mayo frecuencia fue el masculino. No existe relación estadisticamente significativa entre edad o sexo con condición de aceptabilidad.

\section{DISCUSIÓN Y COMENTARIOS}

La población estudiada total fue de 217 pacientes encontrándose como edad más frecuente los 12 años con $12,9 \%$, y una media de 10,09 años, al distribuir los pacientes por grupos etáreos encontramos que el grupo más numeroso corresponde al grupo de 10 a 17 años con $57,14 \%$ y el resto al grupo de 3 a 9 años, esta diferencia parece obedecer a las diferentes actividades físicas que realizan. Al distribuir los casos según el sexo hallamos concordando con la bibliografía de Vals J. $E_{(3)}^{(3)}$, que en su mayoria corresponden al masculino con $79,72 \%$, al parecer debido a su comportamiento en general. Concordando con la bibliografía consultada ${ }^{(2,3,4,9,12)}$ el mecanismo de acción más frecuente que se encontró fue el indirecto con un $87,56 \%$. Del mismo modo el mecanismo indirecto del tipo flexión resulta ser el más frecuente con $72,11 \%$ lo que también esta de acuerdo con los estudios anteriormente citados. Observamos que en forma más frecuente con $67,28 \%$ de los casos los huesos afectados fueron la fractura doble de cúbito y radio, lo que no difiere de la bibliografía revisada. ${ }^{(4)}$ La dirección hallada más frecuentemente se encuentra en los dos planos sagital y coronal correspondientes a la posición anatómica, de ellas la más frecuente en ambos huesos es la varoventral con $53,18 \%$ para el cúbito y $47,37 \%$ para el radio. La extensión de la fractura más frecuente hallada fue la completa y en menor medida la extensión en tallo verde. En e caso particular de la extensión completa el mayor porcen- taje corresponde a la afectación de ambos huesos con $67,49 \%$; y en el caso de la extensión en tallo verde el hueso más comprometido fue el cúbito; estos valores discrepan con la bibliografía que refiere que la extensión en tallo verde es la más frecuente ${ }^{(b)}$, lo que puede deberse a que el número mayor de casos se encuentra en las edades superiores a 10 años. En ambos huesos el trazo hallado con mayor frecuencia corresponde al transversal con $71,10 \%$ en el caso, del cúbito y $71,58 \%$ en el caso del radio, y en segundo lugar el trazo oblicuo en ambos casos. Estos resultados difieren con los textos consultados que indican que el trazo oblicuo es el más frecuente , $^{(3.12)}$ Nuestros resultados en lo que se refieren a la localización de la fractura son concordantes en el orden de frecuencia de afectación con lo que indica Blount, encontrando con mayor frecuencia el trazo en el tercio inferio con $52,02 \%$ para el cúbito y $60 \%$ para el radio, seguido por el tercio medio en ambos huesos con $43,35 \%$ y $34,74 \%$ para el cúbito y radio respectivamente.

Al evaluar las características radiológicas como angulación desplazamiento y cabalgamiento antes de reducción incruenta vimos que: En el cúbito existía una angulación máxima de 40 grados para la incidencia de frente y 35 grados para la de perfil cuyas medias fueron de 11,64 grados y 10,40 grados respectivamente. En e radio encontramos un máximo de 38 grados en la incidencia de frente y de 30 grados para la de perfil y cuyas medias oscilan entre 10,72 grados y 10,09 grados en las incidencias de frente y perfil respectivamente. El desplazamiento varia desde cero hasta $100 \%$ siendo los valores promedio en el caso del cúbito $39.02 \%$ y en el radio $44,08 \%$. Los casos encontrados de cabalgamiento son en menor número, en el caso del cúbito con una frecuencia total de 19 y en el del radio de 29. Asimismo encontrándose un máximo de $15 \mathrm{~mm}$ para el cúbito y 20 para el radio. $\mathrm{Y}$ al evaluar las características radiológicas como angulación desplazamiento y cabalgamiento después de la reducción incruenta vimos que: En la angulación del cúbito, en la incidencia de frente se mostraba una media de 4,7 grados y un máximo de 18 grados; y para la incidencia de perfil una media de 4,2; y un máximo de 25 grados. Al dividir la angulación por grupos observamos que un $96,53 \%$ en la incidencia de frente y un $94,80 \%$ en la incidencia de perfil corresponden al grupo de $\mathrm{O}$ a 10 grados y el grupo de 11 a más grados con un porcentaje minimo. En el caso del radio ambas medias correspondientes a las incidencias de frente y perfil se hallaron por debajo de los 10 grados, encontrando como valor máximo 17 grados en el caso de la incidencia de frente y 24 grados en la de perfil, al realizar la distribución de acuer do a grupos, un alto porcentaje $(92,11 \%$ y $89,47 \%)$ de ambas incidencias correspondieron al grupo de 0 a 10 grados. Resultados concordantes con los valores de re. ducción recomendados por Noonan y Bhaskar.

Al analizar la angulación de acuerdo a hueso afectado y grupo etáreo se observó que en el grupo de 3 a 9 años al afectarse un solo hueso los promedios fueron menores frente a los promedios de afectación de ambos huesos en ambas incidencias, en el grupo de 10 a 17 años los resultados son similares excepto en la incidencia de perfil que son mayores. Aproximadamente en un cuarto de

\section{Situa - XXII}


los casos para ambos huesos $27,75 \%$ para el cúbito y $22,63 \%$ para el radio) el desplazamiento alcanzado luego de la reducción incruenta fue de $0 \%$, el promedio de desplazamiento para ambos huesos logrado luego del tratamiento fue de $21,43 \%$ para el cúbito y $24,83 \%$ para el radio, nuevamente al distribuir los casos por grupos los más altos porcentajes corresponden al grupo de 0 a $50 \%$ ( $89,08 \%$ para el cúbito y $88,42 \%$ para el radio). Como se observa en estos resultados un alto porcentaje se encuentra en los valores aceptables. Cuando hay afectación de un solo hueso observamos en el grupo de 3 a 9 años que las medias son mayores respecto a la afectación de ambos huesos y en el grupo de 10 a 17 años se aprecia el mismo fenómeno excepto para el caso del cúbito en el cual es menor. Al medir el cabalgamiento luego del tratamiento el número de casos encontrados fueron escasos con 15 casos para el cúbito y 17 para el radio, la media hallada fue de $6,27 \mathrm{~mm}$ y el máximo de $15 \mathrm{~mm}$ para el cúbito y en el caso del radio la media calculada fue de $5,06 \mathrm{~mm}$ y el valor máximo de $15 \mathrm{~mm}$. Los casos considerados con de $29,03 \%$ y $39,17 \%$ para los grupos de 3 a 9 años y de 10 a $29,03 \%$ y $39,17 \%$ para los grupos de 3 a 9 años y de 10 a 17 años respectivamente, aunque altos por la suma de ambos grupos llegando aproximadamente al $70 \%$; aparentemente no guardan relación con los resultados anteriores de angulación y desplazamiento analizados por separado ya que estos alcanzan porcentajes cercanos al $90 \%$ de "adecuada reducción" (en el caso de angulación un valor igual o inferior a 10 grados y en el caso del desplazamiento igual o menor al $50 \%$ ),esto es explicable debido a que en cada caso era suficiente que uno de estos valores estuviera por encima, para que fuera considerado como no aceptable.

Realizado el análisis inferencial usando como prueba estadistica a la chi-cuadrado de McNemar no hallamos relación estadisticamente significativa entre edad o sexo y condición de aceptabilidad.

\section{BIBLIOGRAFIA}

1. H. Crenshaw, M.D. CAMPBELL. CIRUGIA ORTOPEDICA. Argentine, novena edición.Ed. Médica Panamericana. 1998.
2. M. O. Tachdjian. ORTOPEDIA PEDIATRICA. México, segunda edición. Ed. Interamericana. 1994: 3388 - 3441.

3. $J$ E. Valls, ORTOPEDIA Y TRAUMATOLOGIA. Argentina, quinta edición, Ed. El Ateneo 1990: 102, 129 - 133.

4. Ramos Vertiz J.R., ELEMENTOS DE TRAUMATOLOGÍA Y ORTOPEDIA, Argentina, quinta edición, Ediciones Cientificas Técnicas Americanas , 1979: 206, 294-296.

5. Bahena V. A. Wata S. G. Gonzales D. "Fracturas de Antebrazo". Revista Hospital Juaréz de México. 2000. Vol 67 Nro 2: 67 - 72.

6. Bhaskar A. R., Roberts J. A. "Treatment of Unstable Fractures of the Forearm in Children" The Journal of Bone and Join Surgery (Br). 2001. Vol 83 - B Nro 2: 253 - 258

7. Tejel M, Prouhasta, "FRACTURAS DE CÚBITO Y RADIO", Sociedad Española de Cirugia Ortopédica y Traumatológica.2000; 8: 15-22.

8. García de Lucas F.,Abad Merenilla J. M., de Porres-Isla Fernández B.,"FRACTURAS DEL RADIO DISTAL", Clínica Fremap, Megadahonda, Madrid, España. 1999;

9. Alkin D.M., Bohay D.R., Slabeuhg D.. Smith B.W. " TREAMENT OF ULNAR SHAFT FRACTURES A PROSPECTIVE RANDOMISED STUDY", Orthopedies, 1995: 18(6) 543-547.

10. Silberman F.,Varaona O.,"ORTOPEDIA Y TRAUMATOLOGIA" Ed. El Ateneo 1995; 239 - 243

11. Rosen P. Doner P. Barkin R, Barkin S,Markovchik V. "DIAG NÓSTICO RADIOLÓGICO EN MEDICINA DE URGENCIA"Ed. Mosby, 1992;171 - 172.

12. Carey PJ, Alburger PD, Betz RR, Clancy M, Steel HH: "BOTH BONE FOREARM FRACTURES IN CHILDREN". Orthopedics 1992; $15: 1015-1019$.

13. Resnick D, Georgen TG, Pathria MN, " HUESOS Y ARTICULACIONES EN IMAGEN", Editorial MARBAN, Segunda edición, pp 721-722.

14. Schwarz, N., Pienaar, S., Schwarz, A. F.,Jelen, M., Styhler, W. Mayr, J, "REFRACTURE OF THE FOREARM IN CHILDREN" British Editorial Society of Bone and Joint Surgery, VOL. 78-B, NO. 5, SEPTEMBER 1996, pp. 740-744

15. Trousdale, R. T. Linscheid, R. L. "OPERATIVE TREATMENT OF MALUNITED FRACTURES OF THE FOREARM", Investigation performed at the Department of Orthopedics, Mayo Clinic and Mayo Foundation, Rochester. The Journal of Bone and Joint Surgery, Incorporated VOL. 77-A, NO. 6, JUNE 1995, pp. 894 902. ( continuan quince más en el trabajo completo)

\section{Internado Rural}

internado rural tiene muchos objetivos, sin embargo la oportunidad que nos brinda para poder servir a lablecicica al poder servir a la población, poder por un mónenta la oportunidad de poder desarrollarse en mejoramiento de los servicios de salud, así como experiencia mas grande que alguna vez he podido experimentar

Int. Adolfo Escalante Portillo 